\title{
Lane consistency of the dispersive coupled-channel optical model potential
}

\author{
R. Capote ${ }^{1, a}$, E. Soukhovitskii ${ }^{2}$, J.M. Quesada ${ }^{3}$, and S. Chiba ${ }^{4}$ \\ 1 Nuclear Data Section, International Atomic Energy Agency, 1400 Vienna, Austria \\ 2 Joint Institute for Energy and Nuclear Research, 220109 Minsk-Sosny, Belarus \\ 3 Departamento de Física Atómica, Molecular y Nuclear, Universidad de Sevilla, 41080 Sevilla, España \\ 4 Advanced Science Research Center, Japan Atomic Energy Agency Tokai, Naka, Ibaraki 319-1195, Japan
}

\begin{abstract}
Recently derived isospin dependent optical model potentials (OMP) containing a dispersive term including non-local contribution are extended to cover 31 actinide nuclei of interest and several Ta- and W-isotopes. As lowlying collective levels of the studied nuclides and Fermi energies are well-known, we only needed to estimate their deformation parameters based on evaluated strength functions. New experimental scattering data are needed to confirm derived regional OMP parameterizations. Isovector components of the dispersive coupled-channel optical model potential for actinides have been challenged on available quasi-elastic $(\mathrm{p}, \mathrm{n})_{I A S}$ scattering data for ${ }^{232} \mathrm{Th}$ and ${ }^{238} \mathrm{U}$ nuclei. Reasonable agreement has been achieved. An exact Lane consistency of the dispersive OMP is discussed. Strict Lane consistency can be achieved by using newly derived formulae and Coulomb corrected energy $\mathrm{E}_{P}-\mathrm{V}_{C}$ in the proton channel.
\end{abstract}

\section{Introduction}

Knowledge of accurate cross sections of a number of reactions (e.g.; total, elastic and inelastic scattering, capture, fission, and so on) between neutrons and actinide nuclei is crucially important for design of various reactor systems. The nuclear reaction data for structural materials like tantalum and tungsten are required to optimize the design of fusion devices like the IFMIF accelerator. The optical model is one of the fundamental theoretical tools which provide the basis of nuclear reaction data analysis and evaluations. The latest extensions of the dispersive coupled-channel optical model (DCCOM) [1] to describe the nucleon interaction on deformed targets, including the quasi-elastic $(\mathrm{p}, \mathrm{n})$ reactions to the isobaric analog state (IAS) of the target ground state are presented.

\section{Dispersive coupled-channel optical model}

Isospin dependent dispersive coupled-channel optical model potentials, which cover the $0.001-200 \mathrm{MeV}$ energy range, have been recently derived both for ${ }^{232} \mathrm{Th}$ and ${ }^{238} \mathrm{U}$ nuclei [2] and W- and Ta-isotopes [3]. The potential of the ref. [2] has been extended to describe ${ }^{233,235} \mathrm{U}$ and ${ }^{239,240,242} \mathrm{Pu}$ isotopes in ref. [4] based on the derived isovector dependence and the observed very weak dependence of the geometrical parameters on mass number.

Following [4] we assumed that geometrical parameters of the optical model potentials depend linearly on mass number $A$ and adjusted only deformation parameters for additional 24 actinides nuclei, and independently for three Ta- and five $\mathrm{W}$-isotopes based on the available strength function information [5,6]. Fitted deformations are in reasonable agreement with FRDM deformations theoretically derived by Möller and Nix [7] as can be observed in table 1 for actinides

${ }^{a}$ Presenting author, e-mail: r.capotenoy@iaea.org and in table 2 for Ta- and $\mathrm{W}$-isotopes (see next page). Those nuclei previously adjusted [2-4] are highlighted in bold. FRDM deformations were used in our calculations for those nuclei where no suitable evaluated strength function data existed.

The Lane consistency of the derived DCCOM potential for actinides has never been challenged on existing IAS scattering data for ${ }^{232} \mathrm{Th}(\mathrm{p}, \mathrm{n})$ and ${ }^{238} \mathrm{U}(\mathrm{p}, \mathrm{n})$ reactions. Therefore, a test of the DCCOM predictive capability for the quasi-elastic $(\mathrm{p}, \mathrm{n})_{\text {IAS }}$ channel is in order. The extent to which we can state that our potential is Lane consistent can be well established from the basic Lane equations [8]:

$$
\begin{aligned}
& V_{p p}=V_{0}+\frac{(N-Z)}{4 A} V_{1} \\
& V_{n n}=V_{0}-\frac{(N-Z)}{4 A} V_{1} \\
& V_{p n}=\frac{\sqrt{(N-Z)}}{2 A} V_{1}
\end{aligned}
$$

where $V_{0}$ and $V_{1}$ are the isoscalar and isovector components of the derived DCCOM potential [2,4] with the Coulomb interaction switched off.

The nuclei studied in this paper are well deformed nuclei characterized by a low-lying rotational band built on top of the ground state. The deformation of these nuclei gives rise to collective motions that result in strong couplings between the low-lying excited states and the ground state and between different excited levels including the isobaric analog states.

An accurate description of the nucleon scattering from these nuclei and of the quasi-elastic $(\mathrm{p}, \mathrm{n})_{\text {IAS }}$ reaction must consider the coupling to the collective inelastic channels including both the ground-state and the IAS rotational bands. A usual way to account for these collective effects is the coupled-channel (CC) method based on Tamura's formalism [9]. The CC code FRESCO [10] was used to perform Lane coupled-channel calculations for the ${ }^{232} \mathrm{Th}(\mathrm{p}, \mathrm{n})$ and ${ }^{238} \mathrm{U}(\mathrm{p}, \mathrm{n})$ reactions using the previously derived DCCOM potential $[2,4]$. No fitting of the potential parameters was 
undertaken. Coulomb corrections as defined in [2,4] were used for a proper description of the charged-particle incident channel. It should be noted that both volume and surface energy-dependent components (including dispersive corrections) contribute to the isovector potential. The coupling $(\mathrm{p}, \mathrm{n})_{I A S}$ form-factors were externally calculated according to

$$
\begin{aligned}
& \left\langle v ; I^{\prime+}(\text { residual })\left|V_{1}(\tau, \vec{r}, E)\right| \pi ; I^{+}(t \arg \text { et })\right\rangle= \\
& \langle v|\tau| \pi\rangle\left\langle I^{\prime}(\text { residual })\left|V_{p n}(\vec{r}, E)\right| I^{+}(t \arg \text { et })\right\rangle= \\
& \frac{\sqrt{N-Z}}{2 A}\left\langle I^{\prime+}(\text { residual })\left|V_{p n}(\vec{r}, E)\right| I^{+}(t \arg \text { et })\right\rangle .
\end{aligned}
$$

In these expressions $\tau$ is the isospin operator, $\boldsymbol{v}$ and $\boldsymbol{\pi}$ represent the entrance and exit isospin states of projectile and ejectile respectively and the isovector coupling potential $V_{p n}(\vec{r}, E)$ should be decomposed into the usual spherical diagonal and deformed non-diagonal coupling components of the potential, as defined in the Tamura's canonical work [9].

Results of the CC calculations for the ${ }^{232} \mathrm{Th}(\mathrm{p}, \mathrm{n}){ }^{232} \mathrm{~Pa}$ reaction at the incident proton energy of $26 \mathrm{MeV}$ are shown in figure 1 in comparison with available experimental data [11,12]. Following Hansen et al. [11] we neglected contributions from the $6^{+}$level in our $(\mathrm{p}, \mathrm{n})$ calculations. Contributions from $\left(0^{+}, 2^{+}, 4^{+}\right)$levels are shown in figure 1 . It is interesting to notice that the calculated isobaric analog cross section to the $2^{+}$level (blue line) above 90 degrees is higher than the cross section corresponding to the isobaric analog ground state (red line); therefore the need for a coupledchannel treatment is demonstrated.

Our calculations show good agreement with the experimental data, especially if we consider that the DCCOM potential was not fitted to those charge-exchange measurements. Similar agreement was obtained for the ${ }^{238} \mathrm{U}(\mathrm{p}, \mathrm{n}){ }^{238} \mathrm{~Np}$ reaction. Therefore we can conclude that our previously derived dispersive coupled-channel for actinide nuclei is also approximately Lane consistent.

\section{Lane consistency of the dispersive potential}

We will discuss in the following subsections the proper requirements to derive a dispersive potential which strictly obeys Lane equations (1).

\subsection{Coulomb Correction (or lack of it)}

It has been previously shown by Wong et al. [13] that, as the energy-independent Coulomb correction $(1.38 \mathrm{Z}) / \mathrm{A}^{1 / 3}$ approximately equals the Coulomb displacement energy $\Delta_{C}$ in a $(p, n)$ reaction, the Lane consistency is only guaranteed for potentials linearly dependent on energy. However, modern dispersive potentials defined in the broad energy range up to $200 \mathrm{MeV}$ are clearly a nonlinear function of energy, so the above mentioned condition is not fulfilled. A closer look to the problem revealed that, as shown by Satchler [14], the proton energy in the expression of the proton potential must be shifted in the nuclear region by the $\Delta_{C}$ to take into account the available kinetic energy.

$$
V_{p p}\left(E_{p}\right)=V_{p p}^{\text {bare }}\left(E_{p}-\Delta_{C}\right) \equiv V_{p p}^{\text {bare }}(E)
$$

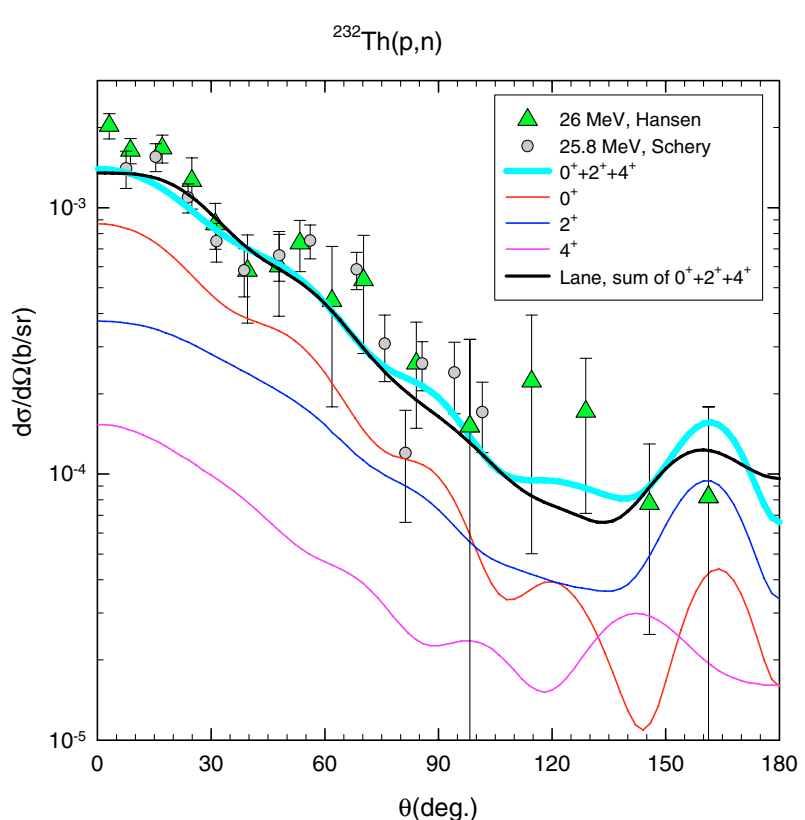

Fig. 1. Dispersive coupled-channel optical model description of the measured $(\mathrm{p}, \mathrm{n})_{I A S}$ angular distribution for ${ }^{232} \mathrm{Th}$ at $26 \mathrm{MeV}$ incident proton energy. The thick cyan line is the sum of the $0^{+}, 2^{+}$and $4^{+}$ CC contributions using DCCOM potential of ref. [2,4]. The bold black line is the sum of the $0^{+}, 2^{+}$and $4^{+} \mathrm{CC}$ contributions for the exact Lane consistent DCCOM potential of this work (see section 3). Experimental data is taken from refs. [11] and [12].

where $\Delta_{C}$ is the Coulomb shift, $E_{n}$ and $E_{p}=E_{n}+\Delta_{C}$ are the incident neutron and proton energies respectively, and the superscript bare refers to the potential with coulomb repulsion switched off and $E$ is the bare nucleon energy. When a Taylor expansion of the right side of the equation (3) is made to the first order, one gets the usual result, namely the Coulomb correction could be calculated as the first derivative in energy of the potential. But if no Taylor expansion is made and instead the energy at which the potential is calculated (i.e. its parameters are fitted) is shifted by the amount $\Delta_{C}$, then there is no need for introducing the Coulomb correction (it is already included to all orders!).

\subsection{Isospin correction term}

According to Mahaux and Sartor [15], the energy at which the proton and neutron potentials are calculated should include an isospin term in the same manner as the Coulomb displacement energy $\Delta_{C}$ for protons was considered in the previous subsection. Namely, the isovector contribution acts as an attractive/repulsive potential for protons and neutrons.

However, only the real part of this term should contribute to this shift in energy as was pointed out by Dabrowski and Sobiczewski $[16,17]$. Following these authors we can write:

$$
\begin{aligned}
& V_{p p}\left(E_{p}-\mu \operatorname{ReV} V_{1}(E)-\Delta_{C}\right) \approx V_{0}(E)-\mu V_{1}(E) \\
& V_{n n}\left(E_{n}+\mu \operatorname{Re} V_{1}(E)\right) \approx V_{0}(E)+\mu V_{1}(E)
\end{aligned}
$$

where $\mu=-\frac{N-Z}{4 A}$ and $V_{0}(E)$ and $V_{1}(E)$ are the isoscalar and isovector components defined at the bare nucleon energy $E$. 
Table 1. Recommended [5,6] and calculatec DCCOM strength functions and $\left(\beta_{2}, \beta_{4}, \beta_{6}\right)$ deformation for 31 actinide nuclei.

\begin{tabular}{|c|c|c|c|c|c|c|c|c|c|c|c|c|}
\hline Nucleus & $\mathrm{S}_{0}$, Minsk & $\mathrm{S}_{0}, \mathrm{RIPL}$ & $\mathrm{S}_{0}$, Calc. & $\mathrm{S}_{1}, \mathrm{RIPL}$ & $\mathrm{S}_{1}$, Calc & $\beta_{2}$, LANL & $\beta_{2}$, Used & $\beta_{4}, \mathrm{LANL}$ & $\beta_{4}$, Used & $\beta_{6}$, LANL & $\beta_{6}$, Used & $x^{2}$ \\
\hline Th-229 & $1.39 \pm 0.69$ & $1.15 \pm 0.15$ & \begin{tabular}{|l|}
0.87 \\
\end{tabular} & - & 2.10 & 0.190 & \begin{tabular}{|l|}
0.178 \\
\end{tabular} & 0.114 & $\begin{array}{l}0.114 \\
\end{array}$ & 0.020 & \begin{tabular}{|l|}
0.020 \\
\end{tabular} & 0.3 \\
\hline Th-230 & $1.47 \pm 0.46$ & $1.28 \pm 0.15$ & 1.03 & - & 2.45 & 0.198 & 0.214 & 0.115 & 0.115 & 0.014 & 0.014 & 1.07 \\
\hline Th-232 & $0.80 \pm 0.08$ & $0.87 \pm 0.07$ & 0.86 & - & 1.72 & 0.207 & 0.213 & 0.108 & 0.069 & 0.003 & 0.0017 & 2.55 \\
\hline $\mathrm{Pa}-231$ & $0.78 \pm 0.35$ & $0.78 \pm 0.08$ & 0.89 & - & 2.59 & 0.198 & 0.178 & 0.115 & 0.115 & 0.012 & 0.012 & 0.04 \\
\hline $\mathrm{Pa}-233$ & $0.80 \pm 0.41$ & $0.75 \pm 0.08$ & 0.97 & - & 2.49 & 0.207 & 0.192 & 0.117 & 0.117 & 0.003 & 0.003 & 0.17 \\
\hline $\mathrm{U}-232$ & $0.88 \pm 0.30$ & $1.4 \pm 0.3$ & 1.10 & - & 1.81 & 0.207 & 0.198 & 0.117 & 0.117 & 0.010 & 0.010 & 1.61 \\
\hline U-233 & $1.07 \pm 0.14$ & $1.04 \pm 0.07$ & 0.93 & - & 2.20 & 0.207 & 0.203 & 0.117 & 0.100 & 0.008 & -.0030 & 0.61 \\
\hline U-234 & $0.81 \pm 0.11$ & $0.85 \pm 0.1$ & 0.77 & - & 2.80 & 0.215 & 0.185 & 0.110 & 0.110 & 0.0 & 0.000 & 0.40 \\
\hline U-235 & $1.01 \pm 0.10$ & $0.88 \pm 0.08$ & 0.93 & $1.8 \pm 0.3$ & 1.93 & 0.215 & 0.211 & 0.110 & 0.107 & -0.005 & -0.021 & 2.05 \\
\hline U-236 & $1.03 \pm 0.13$ & $1.08 \pm 0.1$ & 1.16 & - & 1.53 & 0.215 & 0.222 & 0.102 & 0.102 & -0.008 & -0.008 & 1.57 \\
\hline U-238 & $1.17 \pm 0.13$ & $1.03 \pm 0.08$ & 0.92 & $1.6 \pm 0.4$ & 1.72 & 0.215 & 0.228 & 0.093 & 0.062 & -0.015 & -0.0056 & 2.10 \\
\hline Np-237 & $0.95 \pm 0.08$ & $0.97 \pm 0.07$ & 1.02 & - & 2.33 & 0.215 & 0.204 & 0.102 & 0.102 & -0.009 & -0.009 & 0.72 \\
\hline $\begin{array}{l}\mathrm{Pu}-238 \\
\end{array}$ & $1.29 \pm 0.27$ & $1.3 \pm 0.3$ & 1.27 & - & 1.67 & 0.215 & 0.224 & 0.102 & 0.102 & -0.012 & -0.012 & 3.04 \\
\hline Pu-239 & $1.30 \pm 0.13$ & $1.25 \pm 0.1$ & 1.18 & $2.3 \pm 0.3$ & 2.19 & 0.223 & 0.219 & 0.095 & 0.095 & -0.018 & -0.016 & 2.64 \\
\hline Pu-240 & $1.07 \pm 0.16$ & $1.05 \pm 0.1$ & 1.03 & - & 1.80 & 0.223 & 0.224 & 0.087 & 0.075 & -0.021 & $-\mathbf{0 . 0 0 8 5}$ & 0.38 \\
\hline $\mathrm{Pu}-241$ & $1.07 \pm 0.16$ & $1.23 \pm 0.13$ & 1.11 & - & 1.59 & 0.224 & 0.232 & 0.079 & 0.079 & -0.024 & -0.024 & 2.40 \\
\hline Pu-242 & $0.91 \pm 0.15$ & $0.98 \pm 0.08$ & 0.93 & - & 1.96 & 0.224 & 0.225 & 0.071 & 0.059 & -0.025 & -0.0068 & 0.44 \\
\hline $\mathrm{Pu}-244$ & $1.24 \pm 0.52$ & $0.9 \pm 0.2$ & 0.90 & - & 1.86 & 0.224 & 0.235 & 0.062 & 0.062 & -0.027 & -0.027 & 0.001 \\
\hline Am-241 & $0.90 \pm 0.12$ & $0.88 \pm 0.06$ & 0.89 & - & 2.15 & 0.223 & 0.207 & 0.087 & 0.070 & -0.022 & -0.01 & 0.93 \\
\hline Am- $242^{m}$ & $1.22 \pm 0.25$ & $1.3 \pm 0.3$ & 1.22 & - & 3.33 & 0.224 & 0.293 & 0.079 & 0.079 & -0.026 & -0.026 & 0.002 \\
\hline Am-243 & $0.90 \pm 0.13$ & $0.98 \pm 0.06$ & 0.90 & - & 2.22 & 0.224 & 0.218 & 0.071 & 0.071 & -0.026 & -0.026 & 0.004 \\
\hline $\mathrm{Cm}-242$ & $0.76 \pm 0.40$ & $0.65 \pm 0.15$ & 0.79 & - & 2.00 & 0.224 & 0.189 & 0.079 & 0.079 & -0.024 & -0.024 & 0.08 \\
\hline $\mathrm{Cm}-243$ & $1.05 \pm 0.23$ & $1.5 \pm 0.3$ & 1.06 & - & 2.12 & 0.234 & 0.226 & 0.073 & 0.073 & -0.030 & -0.030 & 0.04 \\
\hline Cm-244 & $1.06 \pm 0.27$ & $1 \pm 0.2$ & 0.98 & - & 1.92 & 0.234 & 0.222 & 0.073 & 0.073 & -0.029 & -.0 .029 & 0.50 \\
\hline Cm-245 & $1.15 \pm 0.19$ & $1.05 \pm 0.15$ & 1.14 & - & 2.38 & 0.234 & 0.246 & 0.064 & 0.064 & -0.031 & -0.031 & 0.004 \\
\hline Cm-246 & $0.91 \pm 0.34$ & $0.45 \pm 0.15$ & 0.98 & - & 2.08 & 0.234 & 0.236 & 0.057 & 0.057 & -0.032 & -0.032 & 0.03 \\
\hline Cm-247 & $0.94 \pm 0.28$ & $0.75 \pm 0.18$ & 0.93 & - & 2.23 & 0.235 & 0.244 & 0.040 & 0.040 & -0.034 & -0.034 & 0.01 \\
\hline Cm-248 & $1.01 \pm 0.24$ & $1.1 \pm 0.12$ & 1.00 & - & 2.34 & 0.235 & 0.252 & 0.040 & 0.040 & -0.036 & -.0 .036 & 0.004 \\
\hline Bk-249 & $1.10 \pm 0.30$ & $1.2 \pm 0.2$ & 1.09 & & 2.38 & 0.235 & 0.257 & 0.040 & 0.040 & -0.037 & -0.037 & 0.002 \\
\hline Cf-249 & $1.03 \pm 0.20$ & $1.2 \pm 0.2$ & 1.03 & & 2.29 & 0.235 & 0.243 & 0.033 & 0.033 & -0.038 & -0.038 & 0.001 \\
\hline Cf-252 & - & - & 0.98 & & 2.54 & 0.236 & 0.252 & 0.015 & 0.015 & -0.035 & -0.035 & 0.08 \\
\hline
\end{tabular}

Table 2. Recommended [5,6] and calculatec DCCOM strength functions and $\left(\beta_{2}, \beta_{4}, \beta_{6}\right)$ deformation for some Ta- and isotopes.

\begin{tabular}{|c|c|c|c|c|c|c|c|c|c|c|c|}
\hline Nucleus & $\mathrm{S}_{0},{ }^{a}$ RIPL & $\mathrm{S}_{0}$, Calc. & $\mathrm{S}_{1}$ RIPL & $\mathrm{S}_{1}$, Calc & $\beta_{2}$, LANL & $\beta_{2}$, Used & $\beta_{4}$, LANL & $\beta_{4}$, Used & $\beta_{6}$, LANL & $\beta_{6}$, Used & $\chi 2$ \\
\hline Ta-180 & $1.9 \pm 0.3$ & 1.63 & - & 0.72 & 0.269 & 0.248 & -0.082 & -0.061 & 0.000 & 0.001 & \\
\hline Ta-181 & $1.7 \pm 0.2$ & $\mathbf{1 . 4 5}$ & - & $\mathbf{0 . 6 9}$ & 0.269 & $\mathbf{0 . 2 4 8}$ & -0.090 & $\mathbf{- 0 . 0 7 1}$ & 0.001 & $\mathbf{- 0 . 0 4 9}$ & $\mathbf{3 . 6 0}$ \\
\hline Ta-182 & $1 \pm 0.5$ & 1.25 & - & 0.68 & 0.259 & 0.259 & -0.101 & -0.101 & 0.005 & 0.005 & \\
\hline $\mathrm{W}-180$ & $1 \pm 0.5$ & 1.74 & - & 0.88 & 0.258 & 0.258 & -0.067 & -0.067 & -0.001 & -0.001 & \\
\hline W-182 & $2.3 \pm 0.3$ & $\mathbf{1 . 7 8}$ & - & $\mathbf{0 . 6 7}$ & 0.259 & $\mathbf{0 . 2 2 9}$ & -0.084 & $-\mathbf{0 . 0 6 4}$ & 0.001 & $\mathbf{0 . 0 0 8}$ & $\mathbf{0 . 9 7}$ \\
\hline W-183 & $1.9 \pm 0.3$ & 1.42 & $0.72 \pm 0.03$ & 0.74 & 0.250 & 0.253 & -0.093 & -0.072 & 0.004 & 0.004 & \\
\hline W-184 & $2.8 \pm 0.4$ & $\mathbf{1 . 5 7}$ & $0.58 \pm 0.07$ & $\mathbf{0 . 6 5}$ & 0.240 & $\mathbf{0 . 2 4 2}$ & -0.095 & $-\mathbf{0 . 0 7 3}$ & 0.010 & $\mathbf{0 . 0 1 7}$ & $\mathbf{1 . 1 5}$ \\
\hline W-186 & $2.1 \pm 0.4$ & $\mathbf{1 . 6 7}$ & $0.37 \pm 0.05$ & $\mathbf{0 . 4 8}$ & 0.230 & $\mathbf{0 . 1 9 9}$ & -0.107 & $\mathbf{- 0 . 0 8 1}$ & 0.020 & $\mathbf{0 . 0 1}$ & $\mathbf{1 . 4 7}$ \\
\hline
\end{tabular}

${ }^{a}$ RIPL data are taken from ref. [5]; Minsk values from ref. [6], LANL deformations from ref. [7].

The assumed Taylor expansion has a high degree of accuracy as the $\mu$ parameter is a very good "small" parameter.

\subsection{Proton and neutron Fermi energies}

Another aspect which must be taken into account to reach the strict Lane consistency is the fact that the Fermi energies are different in the proton and neutron channels. Fortunately we can treat these differences in the same way as previously, namely as a shift $\Delta \varepsilon$ with respect to an average Fermi energy $\left\langle E_{F}\right\rangle$ just by realizing that the Fermi energy always appears in our potentials as a subtracting term to the particle's energy:

$$
\begin{aligned}
& E_{F}^{p}=\left\langle E_{F}\right\rangle-\Delta \varepsilon \\
& E_{F}^{n}=\left\langle E_{F}\right\rangle+\Delta \varepsilon
\end{aligned}
$$

where $\Delta \varepsilon$ is the energy shift associated with the difference of the neutron and proton Fermi energies 


\subsection{Final formulae of the Lane consistency}

Starting from the equation (4) and considering eqs. (3) and (5) we can show [18] that the following relations for dispersive potentials are obtained:

$$
\begin{aligned}
\operatorname{ReV}_{p p}\left(E_{p}-\Delta_{C}-\Delta \varepsilon\right) & \approx \Delta V_{0}(E)-\mu \Delta V_{1}(E) \\
\operatorname{Im} V_{p p}\left(E_{p}-\Delta_{C}-\Delta \varepsilon\right) \approx & \operatorname{Im} V_{0}(E)-\mu \operatorname{Im} V_{1}(E) \\
& +\mu \Delta V_{1}(E) \frac{\frac{d}{d E}\left[\operatorname{Im} V_{0}(E)\right]}{1-\frac{d}{d E}\left[\Delta V_{0}(E)\right]}
\end{aligned}
$$

being $\Delta \mathrm{V}_{0,1}(\mathrm{E})$ the isoscalar/isovector dispersive correction calculated from the corresponding $\operatorname{Im} V_{0,1}(E)$ imaginary potential. Relations (6) and (7) hold both for surface and volume components of the dispersive potentials. Equation (6) is the result that we also obtain for non-dispersive potentials. However, equation (7) contains a new term in the right side which is proportional to $\mu \Delta V_{1}(E)$; being a pure isospin and dispersive correction to the imaginary potential. Authors are not aware of any previous derivation of such contribution. Similar equations are valid for the neutron channel.

We implemented the exact Lane consistent formulation into the OPTMAN code $[18,19]$ and refitted the DCCOM potential parameters using the same experimental database as in refs. [2,4] and also including the available chargeexchange data $(\mathrm{p}, \mathrm{n})_{I A S}$ for both ${ }^{238} \mathrm{U}$ and ${ }^{232} \mathrm{Th}$ nuclei. The new potential will be published elsewhere [18]. The $(\mathrm{p}, \mathrm{n})_{\text {IAS }}$ angular distribution for the ${ }^{232} \mathrm{Th}(\mathrm{p}, \mathrm{n}){ }^{232} \mathrm{~Pa}$ at $26 \mathrm{MeV}$ incident proton energy has been calculated with the new potential and is shown as a bold black line in figure 1. While the observed agreement is similar to the one obtained for the DCCOM potential, we should point out that only two $(p, n)$ measurements were included into our very big nucleon scattering database comprising all available experimental data for actinide nuclei up to $200 \mathrm{MeV}[2,4]$. Therefore the impact of the newly added (p, n) datasets was expected to be small. In fact our calculations are smoother than those shown by Hansen et al. [12], probably because our potential is constrained to reproduce the whole scattering database, while Hansen et al. [12] fitted potential depths to optimise the agreement with measured $(\mathrm{p}, \mathrm{n})_{I A S}$ data around $26 \mathrm{MeV}$.

\section{Conclusions}

Recently derived DCCOM potentials have been extended to cover 31 actinide nuclei of interest and several Ta- and W- isotopes. Isovector components of the dispersive coupled-channel optical model potential for actinides have been challenged on available quasi-elastic $(\mathrm{p}, \mathrm{n})_{I A S}$ scattering data for ${ }^{232} \mathrm{Th}$ and ${ }^{238} \mathrm{U}$ nuclei. Reasonable agreement has been achieved. An exact Lane consistency of the dispersive OMP has been discussed. Strict Lane consistency of the dispersive can be achieved by using newly derived formulae.

\section{References}

1. E.Sh. Soukhovitskii, R. Capote, J.M. Quesada, S. Chiba, Phys. Rev. C 72, 024604 (2005).

2. R. Capote, E.Sh. Soukhovitskii, J.M. Quesada, S. Chiba, Phys. Rev. C 72, 064610 (2005).

3. R. Capote, E.Sh. Soukhovitskii, J.M. Quesada, S. Chiba, Isospin dependent dispersive coupled channel optical model potential for tungsten isotopes, presented at the 11th International Conference on Nuclear Reaction Mechanisms, Varenna, Italy, Villa Monastero, June 12-16, 2006.

4. R. Capote, E.Sh. Soukhovitskii, J.M. Quesada, S. Chiba, Isospin dependent dispersive coupled channel optical model potential for actinides, presented at the Workshop Perspectives on Nuclear Data for the Next Decade at CEA/DIF Bruyères-leChâtel, France, September 26-28, 2005; NEA 6121 report (OECD/NEA, Paris, 2006), p. 43.

5. T. Belgya, O. Bersillon, R. Capote, T. Fukahori, G. Zhigang, S. Goriely et al., Handbook for calculations of nuclear reaction data: Reference Input Parameter Library-2, Technical Report IAEA-TECDOC-1506 (IAEA, Vienna, Austria, 2006), online at www-nds.iaea.org/RIPL2/

6. Yu.V. Porodzinskii, E.Sh. Sukhovitskij, V.M. Maslov, IAEA report INDC(BLR)-012 (Vienna, Austria, 1998).

7. P. Möller, J.R. Nix, W.D. Myers, W.J. Swiatecki, At. Data Nucl. Data Tables 59, 185 (1995).

8. A.M. Lane, Phys. Rev. Lett. 8, 171 (1962).

9. T. Tamura, Rev. Mod. Phys. 37, 679 (1965).

10. I.J. Thompson, Comp. Phys. Rep. 7, 167 (1988).

11. L.F. Hansen, S.M. Grimes, C.H. Poppe, C. Wong, Phys. Rev. C 28, 1934 (1984).

12. S.D. Schery, D.A. Lind, H.W. Fielding, C.D. Zafiratos, Nucl. Phys. A 234, 109 (1974).

13. C. Wong, S.M. Grimes, R.W. Finlay, Phys. Rev. C 29, 1710 (1984).

14. G.R. Satchler, Introduction to Nuclear Reactions (The Macmillan Press Ltd., New York, 1980).

15. C. Mahaux, R. Sartor, Nucl. Phys. A 481, 407 (1988).

16. J. Dabrowski, A. Sobiczewski, Phys. Lett. 5, 87 (1963).

17. J. Dabrowski, Phys. Lett. 8, 90 (1964).

18. J.M. Quesada, R. Capote, E.Sh. Soukhovitskii, S. Chiba (to be published).

19. E.Sh. Soukhovitskii, S. Chiba, O. Iwamoto et al., Programs OPTMAN and SHEMMAN Version 8, Technical report JAERI Data/Code 2005-002 (Japan Atomic Energy Research Institute, Ibaraki, Japan, 2005). 